\section{Effective Cholesterol Management With Fewer Dollars}

Employers, government, and health plans look to managed care pharmacists for ideas and programs to deliver the same or better outcomes at lower cost. Managed care pharmacists can look to the experience at Kaiser in Northern California in 2002 for a combination of methods to improve the efficiency of cholesterol reduction within coronary risk management. Kaiser and its 3 million members in North California use a computerized (electronic) medical record (EMR) and generic lovastatin to achieve superior clinical outcomes at lower cost for members with elevated cholesterol. In advance of the December 2001 introduction of generic lovastatin, Kaiser revised its cholesterol management strategy to start all new patients on generic lovastatin and dose lovastatin to target goal of low-density lipoprotein (LDL); atorvastatin is used only in patients who cannot tolerate lovastatin or cannot reach LDL target goal. Kaiser found that it can treat 5 patients with lovastatin for the same drug cost as just one patient on atorvastatin. ${ }^{1}$ The EMR and clinical practice guideline yield superior clinical results, with $60 \%$ to $70 \%$ of new patients still taking the statin drug at one year compared to the national benchmark of about 30\%. About 85\% of Kaiser Northern California members on a statin drug have LDL cholesterol below $130 \mathrm{mg} / \mathrm{dL}$, nearly twice the national average of $45 \%$ and nearly 4 times Kaiser's 22\% rate in $1997 .^{2}$ The value-for-money interventions at Kaiser extend to the use of generic beta-blockers and generic lisinopril in patients with or at high-risk of heart failure. Kaiser data also appeared to show that longer-term outcomes are superior, as measured by a $30 \%$ lower risk of death due to heart disease among Kaiser members compared to other Californians.

\section{Tablet Splitting to Improve the Value-for-Money} Equation in Cholesterol Management

In a previous issue of the Journal, Calabrese and Baldinger described a dose optimization program using pharmacy claims data to target patients in 15 drug categories to achieve $\$ 1.67$ permember-per-year (PMPY) or $\$ 0.14$ per-member-per-month (PMPM) savings, in 2001 dollars, across the entire population of 234,000 members. ${ }^{3}$ This intervention included 3 statin drugs. In this issue of the Journal, Gee, Hasson, Hahn, and Ryono describe another value-for-money intervention involving tablet splitting of 3 statin drugs. ${ }^{4}$ Conducted prior to the market introduction of generic lovastatin, the researchers found that tablet splitting was associated with favorable clinical (laboratory) outcomes and humanistic-service outcomes while creating the opportunity to treat nearly twice as many patients for the same cost.

This work by Gee, Hasson, Hahn, and Ryono is at least the third published study of tablet-splitting interventions that has found favorable clinical or service outcomes attendant to significant improvement in cost outcomes. There are 2 studies that have measured the effects of tablet splitting on clinical outcomes. As noted by the authors, their work was preceded by a small study that found no significant difference in blood pressure among patients who took whole tablets of lisinopril compared to the same patients who crossed over to split tablets of lisinopril. ${ }^{3}$ A second study was conducted among patients who split tablets of the anticholesterol drugs simvastatin and atorvastatin, 2 of the 3 statin drugs studied by Gee, Hasson, Hahn, and Ryono. As with the current study, there was statistically significant but not clinically significant improvement in intermediate outcomes (i.e., serum lipid levels). ${ }^{4}$ However, unlike the work of Gee, Hasson, Hahn, and Ryono, the earlier study involved a small number of patients $(\mathrm{N}=125)$ and did not measure humanistic outcomes (e.g., service perception or patient satisfaction).

Another point regarding the study of Gee, Hasson, Hahn, and Ryono is remarkable. These authors recognize the important distinction between clinical analysis and statistical analysis. The relatively large study population, 519 patients in their Phase III analysis of laboratory values, contributed to findings of statistical significance for key intermediate clinical outcomes, lipid serum levels, for the tablet-splitting period compared to the prior period. Low-density lipoprotein (LDL) improved to an average $97 \mathrm{mg} / \mathrm{dL}$ in the tablet-splitting period from an average $102 \mathrm{md} / \mathrm{dL}$ prior to tablet splitting, a relative improvement of $5 \%(P<0.001)$. While the authors correctly point out that this finding is not clinically significant, it could be of practical significance since the change could be described and construed as significant improvement according to internal and external reviews of health plan performance for quality-of-care measures. For example, the performance measure for "Cholesterol Management-Control" within the HEDIS (Health Plan and Employer Data Information Set) is defined as "the percentage of health plan members aged 18 to 75 who had evidence of an acute cardiovascular event (hospitalization for acute myocardial infarction, coronary artery bypass graft, or percutaneous transluminal coronary angioplasty) and whose LDL-C was screened and controlled to less than $130 \mathrm{mg} / \mathrm{dL}$ in the year following the event." The Adult Treatment Panel III guidelines of the National Cholesterol Education Program of the National Heart, Lung, and Blood Institute released in May 2001 lowered the ideal LDL level to below $100 \mathrm{mg} / \mathrm{dL}$ for "high-risk" patients. ${ }^{6}$ According to a standard of $100 \mathrm{mg} / \mathrm{dL}$ LDL-C, the tablet-splitting intervention described by Gee, Hasson, Hahn, and Ryono, could make the difference between success and failure in the determination of a "high-performing" health plan and "failure" to manage cholesterol successfully.

\section{Single-Patient Trial (SPT) Method- Substitute for Expert Opinion?}

A remarkable $40 \%$ of patients with gastroesophageal reflux disease (GERD) treated with proton pump inhibitors (PPIs) were found by the single-patient trial (SPT) method to achieve the same or better outcomes as these patients treated with ranitidine. ${ }^{7}$ This work by Wolfe, del Rio, Weiss, et al. suggests that spending on drug treatment for GERD could be reduced by more than one third since histamine-2 receptor antagonists such as ranitidine cost managed care organizations (MCOs) $\$ .50$ or less per day of therapy versus $\$ 4$ or more per day of therapy with a PPI. All of the $\mathrm{H}_{2}$-antagonists (cimetidine, ranitidine, 
famotidine, and nizatidine) were available from generic manufacturers by mid-2002. The availability of generic omeprazole, anticipated at year-end 2002 or early in 2003, would, of course, mitigate the impact of this SPT research on $\mathrm{H}_{2}$-antagonists versus PPIs in 2003 and thereafter. However, the research method itself is intriguing and may have application for other disease conditions.

Readers will note that the authors used the Federal Supply Schedule to make their cost comparison for omeprazole versus generic ranitidine. While the prices in this schedule are not relevant to most MCOs, the relative cost difference is representative of the experience of most MCOs. Actual MCO cost-savings net of copay for the use of generic ranitidine versus (brand) omeprazole would generally exceed $\$ 2.50$ per day of therapy or about $\$ 912.50$ per year, greater than the $\$ 718.44$ absolute savings per patient per year estimated by the authors.

\section{- Paying for Value in the Management of Multiple Sclerosis}

In mid-May 2002, federal health officials announced that Medicare would cover interferon beta-la (Avonex, Biogen) ${ }^{8}$ for multiple sclerosis (MS) but not 3 other commonly prescribed MS disease-modifying medications. The policy change was disclosed in a memo sent to the private health plans that are under contract to process Medicare Part B claims for the government. Effective August 1, 2002, Medicare would pay for injectable drugs that beneficiaries self-administer less than $50 \%$ of the time, according to the Centers for Medicare and Medicaid Services (CMS). The new guidance clarifies Medicare policy for determining whether a drug is "not usually self-administered" and therefore eligible for coverage under federal law. Up to this time, Medicare contractors had interpreted past guidance in varying ways, and, at the time of the announcement, CMS reported that fewer than half of Medicare carriers were paying for Avonex. The logic used by CMS in making the new coverage determination for Avonex but not the other 3 products was that Avonex is injected into muscle, allowing the presumption that it is usually not self-administered, meeting the criteria for Medicare coverage. Rival MS therapies, on the other hand, are not covered because they are administered subcutaneously and could typically be self-administered by the patient. The competing products for treatment of MS at the time of the federal ruling included interferon beta-la (Rebif, Serono SA), glatiramer acetate (Copaxone, Teva Pharmaceutical Industries, Ltd.), and interferon beta-1b (Betaseron, Chiron Corp., marketed by Schering AG's Berlex Laboratories).

There is no cure for MS, and the treatment goal centers on prevention of relapses and retarding progression of the disease. ${ }^{9}$ There are 4 categories of disease: relapsing-remitting (RRMS), secondary progressive (SPMS), primary progressive (PPMS), and progressive relapsing (PRMS). ${ }^{10}$ As with many diseases, MS patients respond selectively to drug therapy. Disease-modifying therapy available today is most effective for the RRMS form, the type of MS most common early in the disease process. ${ }^{11}$ All 3 of the beta interferons and glatiramer are approved by the U.S.
Food and Drug Administration only for the RRMS form of MS. ${ }^{12}$

In this issue of the Journal, Ollendorf, Jilinskaia, and OleenBurkey found a small but measurable advantage in clinical outcomes (i.e., incidence of relapse) and cost outcomes (i.e., drug cost and total direct costs of MS-related care) for glatiramer compared to 2 beta interferon products. ${ }^{13}$ Glatiramer requires daily dosing, while beta- $1 \mathrm{~b}$ is administered every other day, and beta-la (Rebif) is administered subcutaneously 3 times per week. Beta-la (Avonex) is dosed less frequently, just once per week, but is administered intramuscularly. The study by Ollendorf, Jilinskaia, and Oleen-Burkey appears to find some advantage in cost of care with glatiramer, but, interestingly, the cost savings did not stem from less laboratory testing for liver function, thyroid, and complete blood counts, an outcome that might be expected based upon product labeling. However, as the authors note, utilization of laboratory tests was low across all 3 disease-modifying agents in their study.

\section{New Generic and OTC Drugs Provide Opportunities for Drug Benefit Managers}

At year-end 2002, for the first time in history, the same strength and dose of a prescription drug was near approval by the FDA for over-the-counter (OTC) use. This milestone was made more significant by the coincident consideration of not one but 2 blockbuster prescription drugs. A decision from the FDA regarding OTC sale of loratadine $10 \mathrm{mg}$ was expected on or before the end of November 2002, and the FDA was expected to approve omeprazole $20 \mathrm{mg}$ for OTC sale late in 2002 or early in 2003. Both developments are significant. For loratadine, the OTC approval of the $10 \mathrm{mg}$ strength could eliminate the prescription version, because under arcane FDA rules, a prescription drug cannot coexist with an OTC drug of the same dose and strength for the same indication. For omeprazole, the market effects would be presumably different since prescription omeprazole is indicated for gastrointestinal ulcers, gastroesophageal reflux disease (GERD), and "other symptoms associated with GERD,"14 while the OTC version of omeprazole has a narrow, tentative approval for short-term relief of "heartburn." ${ }^{15}$ In the world of high-stakes marketing of prescription drugs, these are unprecedented events. At the same time, Congress was strenuously debating ways to reduce the burden of prescription drug costs for federal programs and the uninsured. The strategic acts by the maker of loratadine and desloratadine could have a negative impact on generic prescription drug competition. Harrington and Shepherd provide a comprehensive review of the regulations and developments in the marketing of OTC drugs that were previously available only by prescription. ${ }^{16}$

Generic omeprazole was still not available to U.S. consumers in October 2002, one year after the patent on omeprazole expired. The manufacturer of omeprazole had reportedly invested 7 years in developing a strategy to negate the market erosion curve expected for omeprazole. ${ }^{17}$ The preferred method involved development of a replacement drug with superior 